\title{
Analysing domestic activity to reduce household energy consumption
}

\author{
Myriam Fréjus ${ }^{\mathrm{a},{ }^{*}}$ and Julien Guibourdenche $\mathrm{e}^{\mathrm{a}, \mathrm{b}}$ \\ ${ }^{a}$ Electricité de France Research and Development, 1 avenue General de Gaulle, 92141 Clamart, France \\ ${ }^{\mathrm{b}}$ Laboratory ICAR (UMR 5191-CNRS), University of Lyon, 5 avenue Pierre Mendes-France, 69500 Bron, France
}

\begin{abstract}
This paper presents our reflections on the issue of behavioral change according to energy conservation constraints and on the status of sustainability in the design of ambient interactive systems at home. We point out how ergonomics contributes to the study of human factors underlying energy consumption. Relating to situated cognition and human computer interaction, our approach relies both on the ergonomic evaluation of feedback consumption displays and on the modeling of domestic activities in order to identify household concerns in real settings. We present empirical results to illustrate this global approach. The results of those studies allow the design of interactive systems: informative and pedagogical systems as well as pervasive and adaptive ambient systems. In our approach, sustainability is taken into account as a design criterion, as security could be, whereas the main design purpose is to aid households in their daily life in order to build a "sustainable situation".
\end{abstract}

Keywords: Models of human activity, sustainable situation, HCI, consumption feedback, sustainability, electricity consumption behavior, situated cognition.

\section{Introduction}

These last decades, many studies on domestic life have been carried out by either academics or industrial research entities [11, 44, 32]. Those studies are driven by research issues, mainly in social sciences, psychology and also ergonomics. They also intend to answer to marketing interests, from competitive companies aimed at developing new products and innovation [46]. Indeed, such studies bring interesting empirical results on the characteristics of domestic activities in everyday contexts. Communication at home, daily routines, privacy issues, technological uses, cooperation and coordination at home are non exhaustive examples of some results.

The growth of domestic studies must be considered in relation with technical developments in the home area (pervasive computing, electricity smart metering) and more generally in relation with the emergence of "smart homes", surrounded by interconnected technologies. Pervasive and information technologies are considered as a way to anticipate and, at least, respond to the needs of the households, thereby working to promote their comfort, convenience, security and entertainment while preserving their natural interaction with the environment [2].

The progressive arrival of ubiquitous computing has fulfilled our domestic environments with new devices, reducing boundaries between the home and the outside [8]. Studying home lives allows the analysis of the existing technologies' uses as well as anticipating the design of new devices. It ensures the development of models of existing practices, through considering the different forms and periods of daily task management as well as the family organization, in relation with the material environment. The design objective is to develop "unremarkable systems" in the sense of [44], i.e. invisible in use, thanks to successful appropriation.

However, studies of home life are also relevant to feed sustainable development issues which have grown up recently as resource preservation and climate change have become a greater preoccupation.

Energy conservation appears as a major concern and leads industrial and academic studies towards different perspectives including human factors underlying

${ }^{*}$ Corresponding author. E-mail: myriam.frejus@edf.fr 
consumption. On that last point, social sciences, psychology and ergonomics are implied in the identification of how consumers take consumption decisions and how people could use energy more wisely [1, 40].

Here prospects for sustainable development meet technological developments, as emerging and existing technology can be a way to aid in reducing consumption [28]. The use of technology can take various forms depending on how it interacts with people at home: displays of information about household consumption, energy management systems to remotely control energy, but also automated and implicit systems to silently control energy. In fact, technology will be used differently according to how consumption reduction is envisioned.

We discuss in the next part how energy savings are generally considered in relation with human practices and technology and we specify our own approach. Then, we give concrete examples of implementation of this approach. This opens several research issues, which are discussed at the end of the article.

\section{Reducing households' energy consumption: study concerns and design purpose}

The mainstream approach is focused on encouraging deliberate reduction behaviors. We will see that it has limitations that have led us to widden our design purpose and consequently redefine our study concern.

\subsection{Changing behavior, incitative and persuasive design}

Encouraging deliberate reduction behaviors implies identifying the underlying factors of behavior in order to influence consumption decisions $[39,7]$. Studies are focused on energy behavior as a subset of domestic behaviors [7]. Values, intention, people's knowledge about energy, personal motivations as well as demographic factors are studied in relation with their consequences on energy use and energy conservation at home.

The purpose is to influence those separate factors to obtain new pro-environmental behaviors at an individual level. Those changes are encouraged either through education, motivation or constraints [37].

Some of those studies are made in relation with design purposes aiming at developing displays for informing consumers of the rates of energy use, and at promoting energy saving actions [49]. The most fre- quent underlying hypothesis is that people will change their behavior if they have proper information and feedback on their actions [31].

A wide range of studies have been published, particularly in the field of human-computer interaction, including the development of different kind of feedback, in relation with the emerging field of persuasive design [3].

However, the feedback effects measured in experiments (see for example the EPRI annual report [15] on energy feedback experiments in smart metering programs) and the results of countries' incentive policies show mild effects on energy consumption [38]. The attempts to influence consumption behaviors rarely cause lasting behavioral changes and do not guarantee the extension of the new behavioral pattern to other domains. Behavior changes are those that have a minor effect on people's lives and not necessarily those who have the most significant impact on the environment [20].

The inertia of household behavior, particularly habitual and automatic, is considered crucial in this failure [19]. The multiplicity of factors involved complicates the search for effective incentives and suggests combining different complementary approaches. If informing people about their consumption is important, it can't be the only resort of influence. But more generally, we assume that the weak results arise because these studies seek only to obtain a pro-environmental intentional behavior and restriction actions. A more or less direct influence between information, intent and behavior is supposed while it hasn't been proven. We believe that the behaviors studied are limited (restricted to energy use and individual actions) and that the study purpose should be extended to take into account other domestic life features. As Strengers points out, "these approaches predominately follow the logic of resource management, rather than domestic life" [40, p.9]. The study purpose extension implies an extension of the design purpose.

\subsection{Informing and designing a sustainable situation}

Rather than directly influencing "the behavior", we believe that a new "situation" might be build that is conducive to both the individual (or the family) and in terms of external evaluation criteria, including the induced level of consumption. Hence the focus is moved from consumption behavior to domestic activities. 
This approach relies on the fact that energy consumption, and especially electricity consumption is a consequence of daily activities and not its purpose [48]. Stern [39] established that energy consumption is not a behavior but the result of behaviors whose purpose, and the contexts in which they occur, do not generally relate to energy savings. Domestic concerns take the form of daily behaviors such as cooking; maintaining one's home; enjoying oneself... In that case, the consumption reduction leans on individual or collective concerns which are not connected with energy or environment.

We assume that daily activity, and more generally human activity, is indeed part of a structural coupling relationship [45] between the actor and the elements of the environment that are selected according to its concerns and experience. Every activity is built on the basis of local interactions in a particular context and in particular material and social circumstances through a process of reciprocal influences between behavior and its context of achievement [29, 41]. We believe that energy-saving solutions can be found by analyzing local interactions between individuals and their environment (this environment is constituted of the family collectively acting out the daily activities in the house). Also the contribution purpose is modified from changing human behavior to assisting daily life, for example with technologies such as ubiquitous computing. We emphasize our ergonomic specificity aiming at designing new situations which help people instead of constraining them. Ergonomics models and methods [43] might help in this perspective, by providing us with empirical knowledge from real life situations.

Hence, in order to achieve energy conservation, two approaches might be privileged:

- Designing an efficient sustainable situation: from the household's point of view (appropriated, with more comfort, ease of use, assisted activities...) and from a sustainable perspective (saving energy). The transformation of the situation with the development of pervasive systems at home could take on some activities to help household while reducing energy consumption. For example, an "intelligent" system which turns off forgotten lights avoids having to come back in the room and also brings about energy reduction. This purpose supposes prior situated analysis of domestic activity to identify people's concerns and needs and to center the design upon a realistic model of individual and collective contexts of activity [24].

- Designing interactive systems displaying feedback on how the evolution of the situation impacted con- sumption: it should enable people to relate their experiences with the underlying consumption. The issue of displaying information is not removed but remains a way to influence and educate in a virtuous circle. The design of feedback systems supposes the prior ergonomic evaluation of existing electricity consumption feedback to find answers to the following questions: what information should be displayed? How could this information be displayed (including its form and the device)?

These two approaches are complementary and imply that sustainable development becomes the concern of the coupling situation between individuals and their environment, instead of, or, waiting for it, to be the direct concerns of behaviors.

The next sections detail our approach by presenting an ergonomic evaluation of consumption feedback systems and an example of analysis of domestic activity in real settings, both in relation with their design implications. These examples relate especially to electricity consumption.

\section{Analysis of the uses of electricity feedback consumption displays: ergonomic evaluation}

We wanted to know what lessons could be learned for our design projects from the numerous experiences already carried out and from existing feedback systems. We carried out a critical analysis of existing studies based upon a literature review and an ergonomic evaluation of existing systems.

\subsection{Literature review}

Numerous works have been published on the issue of informing customers about their electricity consumption via dedicated supports (displays), mainly in the fields of Human Computer Interaction (HCI) and Human Sciences (social psychology and cognitive ergonomics in particular): customer experimentation and evaluations [15], design possibilities and "informative art" [16], dedicated display tests [26], detailed billing tests [30], ambient display tests [35], websites [9], mobile applications tests [18], persuasive games [5], TV [6] and even robots [25], etc. [17]. We therefore felt it was important to capitalize on their results. Two approaches are compared in literature: one approach which involves trying to influence consumption behavior upstream (campaigns in the mass media, general information, customer meetings, home audits...), and another approach which is based on 
feedback on actions (consumption feedback and rewards) [1]. [15] set out six types of consumption feedback, of increasing complexity: standard energy billing, improved billing (information, advice), estimated feedback (estimation of uses, evaluation made on the web, bill analysis), daily / weekly feedback, real-time feedback, improved real-time feedback (by use, control).

Although consumption displays is a constantly changing field, we can see that the functional perimeters which are envisaged, within the constraints imposed by technical possibilities and by the nature of the information concerned, are relatively recurrent and redundant: feedback on past consumption, projection of future consumption, comparisons, general information, advice and incentive, current consumption and current differential tariff plus next switching to another tariff (for real time displays). Consumption display is always based on combinations of functionalities.

Regarding interactive devices, it is relatively difficult to presuppose that any one device will be better than another. We come to the conclusion that it is important to build system complementarity. Numerical display units are part of an overall system of information on consumption, based on several different devices, from the energy bill to the Internet.

The form that these functionalities should take, although crucial, has not often been evaluated from the user point of view while it also influences the efficiency of such devices. [26] thus explains the lack of effectiveness of the devices by the fact that most consumption display systems do not conform to the basic recognized principles of Human Computer Interaction. The in-home positioning of display units vindicates an aesthetic approach which aims to render them more "engaging", but it has to be said that their utility and appropriability for the general public have rarely been examined. The messages being conveyed are very often presupposed by the designers rather than doing any real work on what makes sense to the users.

Also, we wanted to make our own opinion, not about long term and situated effects of feedback, but on their usability and their functional utility from users' points of view.

\subsection{Ergonomic evaluation of real time consumption display systems}

We made an ergonomic evaluation of existing consumption display systems, in order to test the func- tional pertinence of these display devices; allow customers to express needs on concrete grounds, based on a specific use; evaluate how easy it is to understand and use these devices; identify the evolutions required - functional, representational and relating to pertinent physical supports and principles of interaction.

\subsubsection{Method}

The panel for this ergonomic evaluation was made up of 15 people ( 8 men and 7 women), from 25 to 65 years old. We varied the status of occupant, the type of household, the family composition.

The users were placed in a realistic situation of use, in a laboratory, which was set up to look like a sitting room in a home. Consumption displays were connected to different pieces of equipment and when one or more of these appliances was used, realistic data was transmitted to the display devices.

Data on users' activity was gathered in various ways: by observing and filming the users while they played out scenarios, such as: "last week you did a major spring cleaning. You used the washing machine and dryer a lot. Can you find out what you consumed?"; with an interview given at the end of the trial on the perceived utility of the different types of consumption and on the concept of consumption displays and their wishes; with a questionnaire at the end of the evaluation ("getting to know you better").

Several types of device were used for this evaluation: four functional display devices (SPARA, ECO EYE, WATTECO, OWL); a non-functional display device studied using screen copies (CHUMBY); a model of a clock displaying time and consumption, an informative design object (Wattson), a prototype application for mobile phones, made especially for the evaluation.

\subsubsection{Main results}

This evaluation shows that there is a real demand for this type of system, in particular because such realtime information is not currently available anywhere in the country. Customer projections for how these appliances will be used are: tests (via current consumption), consumption monitoring (cumulated consumption) and historical comparison.

Two logics of use would appear to be predominant in relation to consumption display systems: the desire to constantly monitor one's consumption over the long term, and an additional use to carry out tests and experiments when using different appliances. 
Yet whilst the utility of these display devices would seem to be confirmed, they suffer from poor usability. Two types of problem have been observed: difficulties in understanding the information displayed (type of information, location, excess information, modes of display, etc.); Difficulties relating to the buttons and button sequences required to access certain functions. So whilst all of the devices tested have their strengths, none of them really meets customer requirement.

The evaluation pointed out the most popular functions (current consumption, cumulative real monthly and annual consumption, consumption history for previous months and years) and other additional functions receiving positive echoes regarding their utility. On the other hand, some functionalities were clearly rejected either because they were not felt to be useful or because they were difficult to manage.

Regarding the interaction devices, it would appear that the development of a dedicated product such as a display unit, along with that of a telephone application, were the most pertinent. These two supports probably relate to different populations (depending on age and how mobile phones are used) and to different uses. The two applications are therefore complementary.

Whatever the device, it is its ease of use which makes the difference and which will ensure that it is used effectively. What users want most from this type of product is, in fact, simplicity. It is thus important to integrate such ease of use during the conception phase. In addition to organizing the information in a logical manner, it is important to clearly show users the nature of the information displayed, to restrict the amount and types of information on the screen, to proscribe multifunction buttons, and to remove all screen delays.

\subsection{Contributions and limitations}

Both the ergonomic evaluation and the literature review allow guiding the user-centered design of consumption feedback systems on three issues: the logics of use, the relevant functions and the complementarity of the systems. Those elements allow building the utility and the ease of use of future feedback systems, considering their complementarity.

However, our literature review as well as our evaluation doesn't allow to resituate the use of feedback in the flow of householders' activities. The elements of activity collected with the evaluation are limited to the activity of using a digital system. How could the consumption information be introduced in the course of activities that have other purposes?

We need also to define how to implement other forms of energy management which blend energy savings with assisting activities. What devices have to be designed to move towards a "sustainable situation," as we have defined it?

Answering these questions suppose enriching our knowledge on the domestic activity and on the emergence of contextual concerns, at individual and collective levels. That's why this analysis of feedback displays' uses are complemented with an analysis of the domestic activity for the design of ambient systems.

\section{Analysis of domestic activity in real settings}

\subsection{Background and issues}

Models of human activity are prerequisite for building appropriate applications [13] and ambient systems [21]. Exploring the local practices, communications and actions that organize both the home spaces [33] and the contexts from which specific needs might emerge seems pivotal. Studies of domestic life $[12,47,4]$ proposed different conceptual models in relation to their design purpose (e.g., communication media, privacy...). Their definitions always involve artifacts, collective activity and practice.

Artifacts are « tools» for domestic tasks and « resources $\gg$ for the organization of the family $[12,42$, 47]. Hence, if many devices consume energy we may barely design automatic «switch OFF» without paying attention to the context of use. These contexts and uses are not only individual. Artifacts and spaces are at the center of everyday conflicts between individuals [4] and hence, require some coordination and negotiation in the family. A vast problem that has not yet been examined is the large distribution of the family across the house and the transformation of family in the space. As people can move and switch devices or not, which arrangements, which configurations of the family and its artifacts do we observe in real settings?

Previous studies have also underlined the routine nature of domestic activity [e.g, 44]. Ethnomethodology also exhaustively shows that the contextual meaning of actions is reflexive and indexical because it always refers to other previous implicit and hidden events and actions. [41] strongly underlined the human-machine asymmetry in accessing the contextual 
features. This challenges the concepts of « activity recognition » and «context-awareness » implied in many technical perspectives.

Moreover different perspectives [44, 12, 32] already questioned many existing technical models that consider human activities as sequences of targeted actions, executed in the same order with no concurrence or interleaving [23]. The former shows that actions constitute and are embedded in broader sequences. In [44], « knocking at the door» contextually contributes to the accomplishment of «walking to school together». [32] showed that «closing shutters" might be integrated into different larger sequences and preoccupations. Hence, action cannot be strictly associated with a particular concern. In the case of energy management, one might wonder how to investigate these characteristics in order to integrate it during the design process.

Furthermore, household's dynamics of engagement seem to be constituted by multiple lines of these « concerns » or «preoccupations », emerging synchronously $[32,34]$. One person may be practically engaged in the ironing while still watching a TV show and staying aware of children's activities for example. People switch from one concern to another [32], questioning the continuity of a "sequentiality » previously outlined. We wonder to which extent (when in the process, how...) it is necessary to consider the complexity of concerns?

\subsection{Theoretical approach}

Our choice was to model activity at two different complementary scales: individual concerns and socio-material distribution.

Our work on individual concerns is conducted in reference to the course of action empirical research program [43].

Our work on distribution integrates is framed on a more singular and eclectic concepts. These were formerly inspired from previous frameworks such as distributed cognition [27] or interactionism [22]. The concerns are considered as emerging properties of the collective domestic activity, along with the local interactions, the place's dynamic constitutions and electricity consumption.

\subsection{Method}

Five dual-earner households with at least one child participated in this study. The data gathered were (a) continuous video recordings of domestic activity (b) verbalizations during self-confrontation interviews. Each household has been recorded six hours a day, during two week-days and a Saturday. After having agreed, household have been recorded in different rooms with synchronic cameras.

First the data were transcribed with the specific method of multi-score [24]. It allows articulating naturally occurring speech and actions, states of artifacts, physical spaces and interviews' verbalizations. The general organization of each individual course of action was reconstructed with the shortened-story methodology [43] and then displayed on the multiscore frame again to analyze their distribution at several layers.

This methodology was applied to sixty-seven minutes of activity with twelve actors, on four different days in two different families. In order to generalize and evaluate, the first categories and analysis were enhanced with 10 moments, involving the five families, where parents take children to sleep.

\subsection{Results}

The results presented here are voluntarily restricted to periods that consist in "taking children to bed". These examples are relative to the contextual variations of concerns and the physical distribution of activity in the house.

\subsubsection{The contextual variations and concerns}

« Taking children to bed» emerges thirteen times. Due to local interactions, the sequences emerge in various contexts and hence never emerge in fixed order. For example parents may begin with reading a story, then prepare the room and say goodnight. Or they may begin with preparing the room, and reading a story, or not reading a story. Hence, the local interactions' contextual variations affect the temporal organization of concerns. For our design purpose, it means that recognizing an activity from a predefined and fixed set of sequences seems difficult for a system.

The individual activity at home is constituted of multiple synchronic lines of different concerns, whose associations vary a lot. For example, « Taking children to bed » might emerge at the same time as following a TV-show or as prospecting interior items on a magazine, or as ironing, and so on. An ambient system that would recognize activity would have to deal with these multiple lines and associations that form different contexts. 
More positively, understanding the contextual variations of the concerns also allows determining the contextual status of artifacts or « energy wastes ». For example, of the ten moments, six showed evidence of a need for energy management on different devices (television screens and audio speakers, lights, radios) and in different rooms. On the one hand, parents of young children are usually concerned with security when preparing children to sleep. As a consequence, they often left lights $\mathrm{ON}$ in apparently empty spaces such as corridors or rooms, in case children go somewhere dangerous. What might appear as an energy waste is indeed contextual to a useful security concern. On the other hand, parents sometimes leave devices $\mathrm{ON}$ in their empty living-rooms or kitchens while caring for children in bedrooms. It can be considered as energy waste only in that later case and an energy management system should not switch off lights in the former example. However, from the household point of view, it also allows a double economy: parents do not move to switch lights and TV OFF, so they won't have to move to switch it back ON afterward. Comfort here takes the lead. Transforming the situation here could consist in putting an automatic system allowing switching on/off the lights to respond both to the energy waste issue and the economy concern; provided that the situation of waste is correctly qualified.

When parents take children to bed they are sometimes « concerned with » what they are to do after the children sleep: watching TV, reading a book, ironing, resuming diner, preparing for tomorrow... here there is anticipations that's impact the decisions of a smart systems designed to reduce consumptions. Is it acceptable to switch off a TV downstairs when parents are upstairs in the child's room? They may be waiting for some programs such as weather reports, the beginning of a film... here emerges the issue of designing an appropriated interaction: according to the uncertainty of the decision to take (switching off the TV downstairs or not), is an automatic system relevant? Do we allow people validating the choice or deliberately putting the room in an automatic mode? The other media switched $\mathrm{ON}$ at the first floor might be used as a support to allow the parents to follow the beginning of the film until their comeback in the living room. In addition to specifying the situations, the interaction between the pervasive system and the household must be considered as a way to manage the contextual variations of concerns.

The complexity of human concerns makes us doubt that a system would ever « recognize » it. It might be important to reflect upon what to recognize other than
« activity » or « purpose ». What cues might help the system to adapt without «knowing » activity? Our analysis also points towards potential conflicts between criteria (security, comfort and energy savings) making more complex the design for energy savings.

\subsubsection{The distribution of domestic activity}

The distribution of activity in the house also might impact the design and how envisaging relevantly switching OFF or ON.

For example, when parents of one of our families go upstairs and left TV and lights $\mathrm{ON}$ in the empty living-room, the specific activity of caring for children requires an important engagement and interaction between parents and children. Hence parents are barely able to actually follow what is going on in the living-room. This is also confirmed by the noisy environment of the bedroom: people talk and the TV of the bedroom provides sounds. In this context, switching off the living-room's lights and TV would surely be the relevant output of the system. The context is provided by multiple activity and environmental cues that seems to argue for switching off.

However, we also identified distributions such as only one parent goes upstairs for taking children to bed, the other staying in the living room, following the TV show while quickly moving in and out the kitchen. It shows that taking children to bed do not emerge the same way at the scale of collective activity and at the scale of the house. In this case, the context provided by the multiple cues seems to argue for not switching off.

Nevertheless, there are more ambiguous issues resulting from the contextual meaning of concerns and the spatial distribution of people in the home in relation with the output of a system. "Taking children to bed » does not begin by coming in the bedroom. Many times it begins outside the bedrooms, for example when parents and children «negotiate » the organization of the evening, after dinner in the kitchen or in the living room. Preparing a bottle for children mobilizes kitchens for example, not only bedrooms. A clear and fixed association between concerns and rooms seems irrelevant for design. Environmental cues seem to play only as negative definitions in that it allows determining what is not going on for people. This latter assumption is strengthened by our last type of result. We observed that a TV may display programs without being watched by people in the same room, implying that one might switch it OFF. On the contrary, the TV sound often provides accounts in the rooms nearby that allow people following programs 
at a relative physical distance from the living room. This ambiguity leads us also to envisage the potential design of explicit interactions between household and the system, in order to decide whether they want to switch OFF or not.

This later point brings us back to the issue of displays, explicit interactions and energy savings with some new lines that we now wish to discuss.

\section{Discussion}

Cognitive ergonomics and HCI have the opportunity to enhance sustainability in daily life. Their theoretical and methodological backgrounds redefine the issue of energy conservation.

By taking into account the existing constraints of daily life, the ergonomic study of human situated activity allows increase understanding of how behaviors emerge, in connection with the concerns of residents. By comparing different concerns we did not observe any concern such as energy conservation, which stand with other results [10]. However, people in the interviews mention that they sometimes neglect to turn lights off: the empirical investigation enabled us to enlighten the meaning and the emergence of such "forgetfulness" in the houses. Our results suggest that the status of energy consumption regarding cognitive processes, activities and communications would be better conceived of as an effect, a peripheral phenomenon growing with the moment-bymoment accomplishment of the collective activity inside the home.

Through designing a "sustainable situation", and thanks to emerging technologies, we aim to manage this peripheral effect by limiting energy wastage as well as assisting people's activities. The objective is a solution that respects the constraints of the household (alert, provide a mobile interaction system to switch off, switch off automatically in predefined contexts) with new adaptive technologies that also allow non energy oriented functionalities, in order to respond to others criteria such as comfort. But at the same time, the utility of the system is not considered only in relation to the activity but in relation with incidental effects such as energy consumption. We feel that this double-purpose for design will impact our usual user centered design criteria; indeed it supposes to articulate individuals-centered design criteria (utility, usability, acceptability) with collective criteria (efficiency in household chores, coordination...) and with situation-centered criteria (consumption, security). A sustainable situation wishes to respond to those different levels of design criteria. Here emerges the question of the concrete dimensions that must be filled in the design process to achieve a sustainable situation.

These issues are in their infancy, along with our answers. Numerous research issues are opened: within pervasive computing (context-aware systems), HCI (sustainability in the design process, interaction definition) and ergonomics (models of domestic activities, household' contexts definition...). Two additional issues seem particularly salient.

Firstly, the inhabitant's need for energy management implies that a pervasive system should be adapted to the contextual concerns of the individuals. This raises issues relative to the gap between this complex human context and the context of the system based upon an environmental capture [14]. This slight gap is due to the asymmetrical relation between environment as raw material, and situation as experienced environment through the individual's activity. The activity is a complex subject to be understood by a computerized system. The analysis in real settings shows that activity is constantly reoriented according to inter-individual interactions and interactions with the physical environment; families are distributed across multiple scales of physical spaces (floors, rooms, systems of tools, voices, noises) and individual and collective scales of activity are intertwined. Thus how to take into account the inevitable gap between the human contexts and the system context in the design process and in the interaction definition? Which models are needed to design adapted systems? How to arbitrate between conflicting design criteria, such as aid, economy and ecology?

Secondly, according to this gap, the system may be unable to determine the appropriate action to take; thus, designing a context-aware system implies designing the interaction with the user in order to manage uncertainty. It should propose some adaptive modes of interaction (automatic, semi-automatic, implicit/incidental or explicit, on multiple devices...) [36, 13]. It means using interactive media at home to allow the household managing the adaptive systems. This can also be a means to display information about conserved energy: either in relation with the activity as a feedback or in order to influence the user hesitating between different options. So, according to which explicit interaction media will be designed, feedback on energy could also be displayed, in the flow of the activity. The issue of displaying consumption feedback is also reintroduced. More generally, interactive media at home could be used to provide information about "invisible current processes" 
in relation with the current activity: indeed thanks to its global vision at the house level, the system may access information out of reach for an individual and this information should be displayed in a collective awareness purpose. Moreover, dedicated media should also be proposed for a specific use dedicated only to consumption feedback. The ergonomic evaluation of existing displays has shown mistakes that should not be done regarding the choice of media as well as their usability. Thus, the future sustainable situation comprises energy oriented media proposing explicit interaction and different other forms of interaction. Many studies are made in this direction, and ergonomics has its say.

\section{References}

[1] W. Abrahamse, L. Steg, C. Vlek and T. Rothengatter, A Review of Intervention Studies Aimed at Household Energy Conservation, Journal of Environmental Psychology 25 (2005), 273-291.

[2] F. Aldrich, Smart homes: past, present and future, in: Inside the Smart Home, R. Harper, ed., Springer, London, 2003, pp. 17-39.

[3] E. Arroyo, L. Bonanni and T. Selker, Waterbot: exploring feedback and persuasive techniques at the sink, in: Proceedings of the SIGCHI conference on Human factors in computing systems, ACM DL, 2005, pp. 631-639.

[4] L. Baillie, and D. Benyon, Place and technology in the home, Computer Supported Cooperative Work 17 (2008), 227-256.

[5] M. Bång, A. Gustafsson and C. Katzeff, Promoting renewed domestic energy consumption patterns with pervasive learning games, in: International Conference on Persuasive Technology, Springer-Verlag, California, 2007, pp. 55-63.

[6] M. Bång, C. Torstensson and C. Katzeff, The powerhouse: a persuasive computer game designed to raise awareness of domestic energy consumption, in: International Conference on Persuasive Technology, Springer-Verlag, Netherlands, 2006, pp. 123-132.

[7] S. Barr, A. Gilg and N. Ford, The household energy gap: examining the divide between habitual and purchase-related conservation behaviours, Energy Policy 33 (2005), 1425-1444.

[8] G. Bell and P. Dourish, Yesterday's tomorrows: notes on ubiquitous computing's dominant vision, Personal Ubiquitous Computing 11 (2007), 133-143.

[9] R. Benders, R. Kok, H. Moll, G. Wiersma and K. Noorman, New approaches for household energy conservation - In search of personal household energy budgets and energy reduction options, Energy Policy 34 (2006) 3612-3622.

[10]M. Chetty, D. Tran and R. Grinter, Getting to Green: Understanding Resource Consumption in the Home, in: UbiComp '08: Proceedings of the 10th International Conference on Ubiquitous Computing, ACM DL, Korea, 2008, pp. 242-251.

[11]A. Crabtree and T. Rodden, Technology and the home: supporting cooperative analysis of the design space, in: CHI'02 « New technologies for families » Workshop, ACM DL, Minnesota, 2002.

[12] A. Crabtree and T. Rodden, Domestic Routines and Design for the Home, Computer Supported Cooperative Work, 13 (2004), 191-220.
[13]A. Dix, Beyond intention - Pushing boundaries with incidental interaction, in: Proceedings of Building Bridges: Interdisciplinary Context-Sensitive Computing, Glasgow, 2002.

[14] M. Dominici, M. Fréjus, J. Guibourdenche, B. Pietropaoli and F. Weis, Towards a System Architecture for Recognizing Domestic Activity by Leveraging a Naturalistic Human Activity Model, in: ICAPS'11 « GAPRec » Workshop, Freiburg, 2011.

[15]Electric Power Research Institute, Residential Electricity Use Feedback: A Research Synthesis and Economic Framework. Palo Alto, CA, 2009.

[16]A. Fercher, M. Hitz and G. Leitner, Pervasive approaches to awareness of energy consumption, in: AmI-Blocks'08, Second European Workshop on Smart Products: Building Blocks of Ambient Intelligence, 2008.

[17]J. Froehlich, L. Findlater and J. Landay, The Design of EcoFeedback Technology. CHI 2010, April 10-15, Atlanta, Georgia, USA.

[18] J. Froehlich, T. Dillahunt, P. Klasnja, J. Mankoff, S. Consolvo, B. Harrison and J. Landay, UbiGreen: Investigating a Mobile Tool for Tracking and Supporting Green Transportation Habits, in: Proceedings of the International Conference on Human Factors in Computing Systems, ACM DL, Boston, 2009, pp. 1043-1052.

[19]G.T. Gardner and P.C. Stern, Environmental problems and human behavior, Boston: Allyn and Bacon, 1996.

[20]B. Gatersleben, L. Steg and C. Vlek, Measurement and determinants of environmentally significant consumer behavior Environment and Behavior 34(2002), 335-362.

[21] S. Greenberg, Context as a dynamic construct, Human Computer Interaction 16 (2001), 257-268

[22]M. Grosjean, From multi-participant talk to genuine polylogue: shift-change briefing sessions at the hospital, The Journal of Pragmatics 36 (2004), 25-52.

[23]T. Gu, Z. Wu, X. Tao, H.K. Pung and J. Lu, Ep-SICAR: An Emerging Patterns Based Approach to Sequential, Interleaved and Concurrent Activity Recognition, in: Proceedings of the International Conference on Pervasive Computing and Communications, IEEE DL, Texas, 2009, pp. 1-9.

[24] J. Guibourdenche, J. Vacherand-Revel, M. Grosjean, M. Fréjus and Y. Haradji, Transcribing the activity of a distributed family, in: Proceedings of the International Conference on Computer Supported Cooperative Work, ACM DL, China, 2011, pp. 637-640.

[25] J. Ham and C. Midden, A Persuasive Robotic Agent to Save Energy: The Influence of Social Feedback, Feedback Valence and Task Similarity on Energy Conservation Behavior. ICSR (2010), 335-344.

[26] E. Hinterbichler, Designing a better energy consumption indicator Interface for the home, Thesis for the degree of Master of Science in Computer Science, University of Illinois at Urbana-Champaign, 2008.

[27]E. Hutchins, Enaction, Imagination and Insight, in: J. Stewart, O. Gapenne \& E. A. Di Paolo, eds, Enaction. Toward a New Paradigm for Cognitive Science, MIT Press, Cambridge, 2010, pp. 425-450.

[28]M. Kaptein, P. Markopoulos, B. De Ruyter and E. Aarts, Persuasion in ambient intelligence, Journal of Ambient Intellingence Humanized Computing 1(2010), 43-56.

[29] J. Lave, Cognition in practice. Cambridge University Press, 1988.

[30]D. Lord, W. Kempton, S. Rashkin, A. Wilson, C. Egan, A. Eide, M. Lyer and C. Payne, Energy Star Billing: Innovative Billing Options for the Residential Sector, Environmental Protection Agency, 1996. 
[31]S. Oskamp, Environmentally responsible behavior: teaching and promoting it effectively, Analysis of Social Issues and $\mathrm{Pu}-$ blic Policy 2(2002), 173-182.

[32]G. Poizat, M. Fréjus and Y. Haradji, Analysis of collective activity in domestic settings for the design ubiquitous technologies, in: Proceedings of the European Conference on Cognitive Ergonomics, ACM DL, Finland, 2009.

[33] M. Relieu, M. Zouinar and N. La Valle, At home with video cameras, Home Cultures, 4(2007), 1-26.

[34]P. Salembier, J. Dugdale, M. Fréjus and Y. Haradji, A descriptive model of contextual activities for the design of domestic situations, in: Proceedings of the European Conference on Cognitive Ergonomics, ACM DL, Finland, 2009.

[35] J. Sauer, C. Schmeink and D.G. Wastell, Feedback quality and environmentally friendly use of domestic central heating systems, Ergonomics 50 (2007), 795-813.

[36]A. Schmidt, M. Kranz and P. Holleis, Interacting with the Ubiquitous Computer Towards Embedding Interaction, in: Proceedings of the Joint sOc-EUSAI Conference, ACM DL, France, 2005, pp. 147-152.

[37]E. Shove, Changing human behaviour and lifestyle: a challenge for sustainable consumption?, in: Consumption - Perspectives from ecological economics, I. Ropke and L. Reisch, eds, Elgar, Cheltenham, 2005, pp. 111-132.

[38]H. Staats, P. Harland and H. Wilke, Effecting durable change: a team approach to improve environmental behavior in the household, Environment and Behavior 36(2004), 341-367.

[39]P.C Stern, What Psychology knows about energy conservation. American Psychologist 47 (1992), 1224-1232.

[40]Y. Strengers, Smart Metering Demand Management Programs: Challenging the Comfort and Cleanliness Habitus of Households, in: Proceedings of the 20th Australasian Conference on Computer-Human Interaction: Designing for Habitus and Habitat, ACM DL, Australia, 2008, pp. 9-16.
[41]L. Suchman, Human-Machine Reconfigurations, Cambridge University Press, New York, 2007.

[42]A. Taylor and L. Swan, Artful Systems in the Home, in: Proceedings of the International Conference on Human Factors in Computing Systems, ACM DL, Oregon, 2005, pp. 641-650.

[43]J. Theureau, Course-of-Action Analysis and Course-ofAction-Centered Design, in: Cognitive Task Design, E. Hollnagel, ed, Erlbaum, London, 2003, pp. 55-81.

[44] P. Tolmie, J. Pycock, T. Diggins, A. MacLean and A. Karsenty, Unremarkable Computing, in: Proceedings of the International Conference on Human Factors in Computing Systems, ACM DL, Minnesota, 2002, 399-406.

[45]F. Varela, E. Thompson and E. Rosch, The Embodied Mind: Cognitive Science and Human Experience, MIT Press, Cambridge, 1991.

[46]A. Venkatesh, A Conceptualisation of the Household/Technology Interaction, Advances in Consumer Research 12 (1985), 189-194.

[47]A. Venkatesh, S. Chen and V. Gonzalez, Designing The Family Portal for Home Networking, in: Domesticating Information Technologies, R. Kraut, M. Brynin and S. Kiesler, eds, Oxford University Press, Oxford, 2005, pp. 109-127.

[48]A. Warde, Consumption and theories of practice, Journal of Consumer Culture 5 (2005), 131-153.

[49] G. Wood and M. Newborough, Energy-use Information Transfer for Intelligent Homes, Energy and Buildings 39 (2007), 495-503. 